\title{
Gilles de la Tourette syndrome
}

\author{
Lt Col RK Saini*, Col J Debnath ${ }^{\dagger}$, Lt Col SK Sahoo ${ }^{\#}$, Col R Goger**
}

MJAFI 2011;67:388-389

\section{INTRODUCTION}

Gilles de la Tourette syndrome is a neuropsychiatric disorder, characterised by involuntary, stereotyped motor and vocal tics of variable severity. ${ }^{1}$ The children suffering from Tourette syndrome (TS) commonly suffer from social isolation and often subjected to abuse by parents and teachers during the school days. Such an approach leads to many emotional problems. ${ }^{2}$ We present such a case wherein a young girl underwent severe emotional trauma after onset of this illness and how the treatment made a change in her life.

\section{CASE REPORT}

A 12-year-old girl was brought by her mother with complaints of making guttural noises and intermittently vocalising bad words or abuses, shrugging her shoulders off and on purposelessly, deteriorating academic performance, and remaining socially withdrawn for the last one year. She was apparently well about one year back with average school performance. She had a bout of sore throat with fever from which she recovered without much complication within 10 days. After few days of the illness she started making guttural noises, vocalising bad words/abuses at inappropriate places and time. She was also noted to be shrugging her shoulders intermittently without any specific purpose. She was often reprimanded for blurting out obscenities and making guttural noises especially while she was in her class or at home close to her mother. However, if she tried to control her symptoms, it led to anxiety, restlessness, and rebound of symptoms. The symptoms caused marked social embarrassment. She started repeatedly rubbing off her writing in her text book. She believed that it was not properly aligned or was not neat and spent hours on single page. It led to significant slowing of her writing. If she did not erase off the text, she felt compelled to "correct" it. Due to the above problem she could not write the class tests and had to be withdrawn from

*Classified Specialist (Psychiatry), 'Senior Advisor (Radio-Diagnosis), 167 Military Hospital, C/o 56 APO, " Graded Specialist (Paediatrics), Military Hospital, Devlali, **Senior Advisor (Psychiatry), Base Hospital, Delhi Cantt. - 10.

Correspondence: Lt Col RK Saini, Classified Specialist (Psychiatry), 167 Military Hospital, C/o 56 APO.

E-mail: rajiv_100_98@yahoo.com

Received: 26.05.2010; Accepted: 20.02.2011

doi: 10.1016/S0377-1237(11)60086-5 school. Then she started remaining sad, socially withdrawn, speaking less, and often cried to self. Subsequently, she manifested pain in the abdomen, headache, and nocturnal enuresis. The symptoms progressed gradually and she was referred to the psychiatrist after the general physician failed to find any organic cause for her symptoms. There was no history of abuse, seizure, syncope, hyperactivity or learning disorder. She had a nine-year-old younger brother who was normal. Her early childhood was uneventful. She was immunised as per schedule and had achieved motor, social, and cognitive milestones according to her age. General physical and systemic examination revealed no abnormality. There were no neurocutaneous markers or thyromegaly. Moreover, ENT examination was normal. Mental status examination revealed a gloomy, withdrawn, and reticent child who made guttural noises intermittently. She shrugged her shoulders intermittently without any purpose. Retrospective analysis of her speech sample (as brought out by the mother) revealed coprolalia. Her mood was depressed. She denied suicidal thoughts, hopelessness or worthlessness. No psychotic features were noted. Her sensorium was clear but biodrives were reduced. Her school notebook showed torn pages and had evidence of repeated rubbing and writing (indicating obsession of neatness, alignment, and compulsion of rubbing and rewriting). All relevant haemato-biochemical parameters, throat swab culture, anti-streptolysin O (ASO) titres, and computed tomography scan of head were normal. With the above information she was diagnosed as a case of Gilles de la Tourette's disorder with obsessive compulsive disorder (OCD) and treated with tab risperidone $1 \mathrm{mg}$ HS, tab clomipramine $50 \mathrm{mg}$ HS besides psychosocial rehabilitation, family, and teacher counselling. She started showing improvement by 10 weeks with gradual reduction of guttural noises, purposeless shrugging of shoulders, coprolalia (which almost disappeared), enuresis, and depressive features. Obsessive symptoms were, however, resistant to these measures. Since her teacher understood her problem after counselling, she was allowed to attend the school. At the end of six months of treatment, her vocal and motor tics of shrugging of shoulders were well controlled though obsessive symptoms were still prominent. She continued to have difficulty in writing rapidly but was able to overcome her disability with good performance in oral tests. As per her mother the child had regained her old self.

\section{DISCUSSION}

Diagnosis of TS is based on the presence of both motor and vocal and motor tics for at least one year. A tic is an involuntary, 
rapid, recurrent, non-rhythmic motor movement or vocal production that is of sudden onset and serves no apparent purpose. Though presence of both is essential for the diagnosis, they need not be present throughout and concurrently. Common forms of tics are facial tics, eye blinking, shrugging of the shoulders, lip smacking, and throat clearing. Sometimes tics could be complex in the form of making thrusting pelvic movements or making certain vocalisations. The diagnosis is purely clinical and there are no laboratory tests to confirm the diagnosis. People with tics often try to control their tics but experience a build-up of an urge or an uncomfortable sensation which gets relieved following the tic. This urge or uncomfortable sensation can take many forms, including an itch, a feeling of tightness, tingling or an experience of irritation or worry. Exact cause of TS is not known though literature points towards an autosomal dominant pattern of inheritance suggesting genetic basis of the illness. ${ }^{3}$ Other factors like Streptococcus infection, magnesium deficiency, low birth weight, and maternal life stress during pregnancy may also precipitate the illness. Tics result from biochemical transmission abnormality in the basal ganglia. The transmitters implicated are dopamine, serotonin, and norepinephrine. ${ }^{4}$ Though the exact incidence is unknown, it is presumed to be $0.022 \%$ in girls and $0.077 \%$ in boys. Males are twice more likely to suffer than females from this illness. ${ }^{5}$ Tics worsen with stress and get better when the person is relaxed or in an activity. Tics reduce or disappear during sleep. Tics eventually fade away with age in most cases. However, some patients with TS can have severe associated emotional problems like self-injurious behaviour, OCD or depression. ${ }^{2}$ Association of TS with OCD is well known as has been the case of Dr Samuel Johnson, who wrote "A dictionary of English language." ${ }^{\circ}$ Our case also highlighted the co-morbidity of TS and OCD. Common drugs to control tics are antipsychotics like haloperidol, risperidone, ziprasidone, pimozide, and fluphenazine. ${ }^{4}$ TS associated emotional problems can be handled with psychotherapy and antidepressant drugs. Patients of TS need a tolerant and encouraging environment and want to be treated as everyone else and others need to be more tolerant and accepting.

\section{REFERENCES}

1. National Institute of Neurological Disorders and Stroke. Tourette Syndrome Fact Sheet, NIH publication no. 05-2163, Publication date April 2005.

2. Schhill L, Williams S, Schwab Stone M, Applegate J, Leckman JF. Disruptive behavior problems in a community sample of children with tic disorders. Adv Neurol 2006;99:184-190.

3. Swain JE, Scahill L, Lombroso PJ, King RA, Leckman JF. Tourette syndrome and tic disorders: a decade of progress. J Am Acad Child Adolesc Psychiatry 2007;46:947-968.

4. Sallee FR, Gilbert DL, Vinks AA, Miceli JJ, Robarge L, Wilner K. Pharmacodynamics of ziprasidone in children and adolescents: impact on dopamine transmission. J Am Acad Child Adolesc Psychiatry 2003; 42:902-907.

5. Zinner SH. Tourette syndrome-much more than tics. Contemp Pediatr 2004;21:22-49.

6. Hood KK, Baptista-Neto L, Beasley PJ, Lobis R, Pravdova I. Case study: severe self-injurious behavior in comorbid Tourette's disorder and OCD. J Am Acad Child Adolesc Psychiatry 2004;43:1298-1303. 